\title{
Light-cone analysis of the pure spinor formalism for the superstring
}

\section{Nathan Berkovits and Renann Lipinski Jusinskas}

ICTP South American Institute for Fundamental Research, Instituto de Física Teórica, UNESP

- Univ. Estadual Paulista, Rua Dr. Bento T. Ferraz 271, 01140-070, São Paulo, SP, Brasil

E-mail: nberkovi@ift.unesp.br, renannlj@ift.unesp.br

ABSTRACT: Physical states of the superstring can be described in light-cone gauge by acting with transverse bosonic $\alpha_{-n}^{j}$ and fermionic $\bar{q}_{-n}^{\dot{a}}$ operators on an $\mathrm{SO}(8)$-covariant superfield where $j, \dot{a}=1$ to 8 . In the pure spinor formalism, these states are described in an $\mathrm{SO}(9,1)$-covariant manner by the cohomology of the BRST charge $Q=\frac{1}{2 \pi i} \oint \lambda^{\alpha} d_{\alpha}$. In this paper, a similarity transformation is found which simplifies the form of $Q$ and maps the light-cone description of the superstring vertices into DDF-like operators in the cohomology of $Q$.

KEYwORDS: Superstrings and Heterotic Strings, BRST Symmetry

ARXIV EPRINT: 1406.2290 


\section{Contents}

1 Introduction 1

2 Superparticle 3

2.1 Review of the pure spinor superparticle 3

2.2 Similarity transformation 4

2.3 Relation with light-cone vertex operators 5

3 Superstring $\quad 6$

$\begin{array}{lll}3.1 & \text { Review of the pure spinor superstring } & 7\end{array}$

$\begin{array}{lll}3.2 & \text { Similarity transformation } & 9\end{array}$

$\begin{array}{lll}3.3 & \text { Relation with light-cone vertex operators } & 10\end{array}$

4 Conclusion $\quad 12$

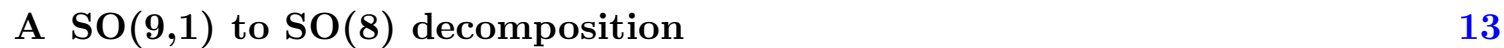

\section{Introduction}

Although the covariant description of string theory is convenient for amplitude computations and for describing curved backgrounds, the light-cone description is convenient for computing the physical spectrum and for proving unitarity. For the manifestly spacetime supersymmetric string, the light-cone description was worked out over 30 years ago [1] but the covariant description using the pure spinor formalism [2] is still being developed.

In this paper, the relation between this covariant and light-cone superstring descriptions will be analyzed. As in other string theories, physical states in the pure spinor formalism are covariantly described by the cohomology of a nilpotent BRST operator. However, because the pure spinor worldsheet ghost is constrained, evaluation of the BRST cohomology is not straightforward. By partially solving the pure spinor constraint, it was proven in [3] that the BRST cohomology reproduces the correct light-cone spectrum. However, the proof was complicated and involved an infinite set of ghosts-for-ghosts. In this paper, the proof will be simplified considerably and an explicit similarity transformation will be given for mapping light-cone superstring vertex operators constructed from the $\mathrm{SO}(8)$-covariant superfields of [4] into DDF-like vertex operators in the cohomology of the pure spinor BRST operator.

In bosonic string theory, the covariant BRST operator

$$
Q_{B}=-\frac{1}{2 \pi i} \oint\left\{\frac{1}{2} c \partial X^{m} \partial X_{m}+i b c \partial c\right\}
$$


can be mapped by a similarity transformation $R$ to the operator

$$
\hat{Q}_{B}=e^{R} Q_{B} e^{-R}=-k^{+} \sum_{n \neq 0} c_{-n} \alpha_{n}^{-}+c_{0}\left(\frac{k^{m} k_{m}}{2}+\sum_{n \neq 0} \alpha_{-n}^{i} \alpha_{n}^{i}-1\right),
$$

where $\alpha_{n}^{-}, \alpha_{n}^{i}$ and $c_{n}$ are modes of the $X^{-}, X^{i}$ and $c$ variables, and $k^{m}$ is the momentum, with $\sqrt{2} k^{+}=\left(k^{0}+k^{d-1}\right)$ assumed to be non-vanishing. Because of the quartet argument, the cohomology of $\hat{Q}_{B}$ is independent of the $\left(c_{n}, b_{n}, \alpha_{n}^{+}, \alpha_{n}^{-}\right)$modes, so the physical spectrum is the usual light-cone spectrum constructed by acting with the transverse $\alpha_{-n}^{i}$ modes on the tachyonic ground state. Furthermore, the similarity transformation $R$ maps light-cone vertex operators $V_{\mathrm{LC}}$ which only depend on the transverse variables into the physical DDF vertex operators $V_{D D F}=e^{-R} V_{L C} e^{R}$ which are in the cohomology of $Q_{B}$ [5].

In the pure spinor formalism, the covariant BRST operator is

$$
Q=\frac{1}{2 \pi i} \oint\left(\lambda^{\alpha} d_{\alpha}\right)
$$

where $\alpha=1$ to 16 is an $\mathrm{SO}(9,1)$ spinor index, $\lambda^{\alpha}$ is a bosonic spinor ghost satisfying the pure spinor constraint

$$
\lambda^{\alpha} \gamma_{\alpha \beta}^{m} \lambda^{\beta}=0,
$$

and $d_{\alpha}$ is the Green-Schwarz-Siegel fermionic constraint which has 8 first-class and 8 secondclass components. A similarity transformation $R$ will be found which maps $Q$ into

$$
\hat{Q}=e^{R} Q e^{-R}=\frac{1}{2 \pi i} \oint\left[\lambda^{a}\left(p_{a}+\frac{\sqrt{2}}{2} \hat{T} \theta_{a}\right)+\bar{\lambda}^{\dot{a}}\left(\bar{p}_{\dot{a}}+\frac{\sqrt{2}}{2} \partial X^{+} \bar{\theta}_{\dot{a}}\right)\right],
$$

where $\theta^{\alpha}=\left(\theta^{a}, \bar{\theta}^{\dot{a}}\right)$ and $p_{\alpha}=\left(p_{a}, \bar{p}_{\dot{a}}\right)$, and $a(\dot{a})$ represent the chiral (antichiral) $\mathrm{SO}(8)$ spinor indices. $\hat{T}$ will include part of the energy-momentum tensor and will impose the usual Virasoro-like conditions.

The cohomology of $\hat{Q}$ will be argued to consist of states which are independent of $\left(\theta^{a}, p_{a}\right)$ and which are constructed from the $\mathrm{SO}(8)$-covariant light-cone superfields $f^{a}(\bar{\theta}) e^{i k \cdot X}$ of $[4]$ by hitting with the transverse raising operators $\alpha_{-n}^{j}$ (bosonic) and $\bar{q}_{-n}^{\dot{a}}$ (fermionic) as

$$
V_{\mathrm{LC}}=\prod_{n, j, \dot{a}}\left(\alpha_{-n}^{j}\right)^{N_{n, j}}\left(\bar{q}_{-n}^{\dot{a}}\right)^{N_{n, \dot{a}}} \lambda^{a} f_{a}(\bar{\theta}) e^{i k \cdot X}
$$

where $k^{m} k_{m}=-2 \sum n\left(N_{n, i}+N_{n, \dot{a}}\right)$. Furthermore, it will be shown that the similarity transformation $R$ maps the light-cone vertex operators of (1.6) into DDF-like vertex operators $V_{D D F}=e^{-R} V_{L C} e^{R}$, which are in the cohomology of the pure spinor BRST operator and which will be described in a separate paper by one of the authors [6]. DDF-like vertex operators in the pure spinor formalism were first constructed by Mukhopadhyay [7] using a Wess-Zumino-like gauge choice which breaks manifest supersymmetry, whereas the recent construction of Jusinskas [6] uses a supersymmetric gauge choice which simplifies the analysis and enables an explicit $\mathrm{SO}(8)$ superfield description of the whole physical spectrum. 
In section 2, the superparticle will be discussed and a similarity transformation $R$ will be constructed which maps the superparticle BRST operator into a simple quadratic form and maps the light-cone $\mathrm{SO}(8)$-covariant superfield of [4] into the super-Yang-Mills vertex operator in the pure spinor formalism. In section 3 , this construction will be generalized to the superstring such that the similarity transformation maps the light-cone vertex operators of (1.6) into the DDF-like vertex operators of [6] in the cohomology of the pure spinor BRST operator.

\section{Superparticle}

In this section, the pure spinor formulation of the superparticle will first be reviewed and a similarity transformation will then be presented which makes the massless constraint explicit in the BRST operator and maps the super-Yang-Mills vertex operator into the light-cone $\mathrm{SO}(8)$ superfield of [4].

\subsection{Review of the pure spinor superparticle}

The pure spinor superparticle was extensively discussed in [8] and is described by the first order action

$$
S=\int d \tau\left\{\dot{X}_{m} P^{m}-\frac{1}{2} P^{m} P_{m}+\dot{\lambda}^{\alpha} \omega_{\alpha}-i \dot{\theta}^{\alpha} p_{\alpha}\right\},
$$

containing the pure spinor ghost $\lambda^{\alpha}$ and the anti-ghost $\omega_{\alpha}$. Note that the dot above the fields represent derivatives with respect to $\tau$.

The BRST charge is defined as

$$
Q=\lambda^{\alpha} d_{\alpha}
$$

where

$$
d_{\alpha}=p_{\alpha}-\frac{i}{2} P_{m}\left(\gamma^{m} \theta\right)_{\alpha}
$$

is the supersymmetric derivative, and the supersymmetry generators are

$$
q_{\alpha}=p_{\alpha}+\frac{i}{2} P_{m}\left(\gamma^{m} \theta\right)_{\alpha} .
$$

Canonical quantization of (2.1) gives

$$
\begin{aligned}
{\left[X^{m}, P^{n}\right] } & =i \eta^{m n}, \\
\left\{\theta^{\alpha}, p_{\beta}\right\} & =i \delta_{\beta}^{\alpha} .
\end{aligned}
$$

Note that $\left\{q_{\alpha}, d_{\beta}\right\}=\left[q_{\alpha}, P_{m}\right]=0$ and $\left\{d_{\alpha}, d_{\beta}\right\}=P_{m} \gamma_{\alpha \beta}^{m}$.

To compare this covariant description with the light-cone description, chiral and antichiral $\mathrm{SO}(9,1)$ spinors will be decomposed into their $\mathrm{SO}(8)$ components as $\theta^{\alpha} \rightarrow\left(\theta^{a}, \bar{\theta}^{\dot{a}}\right)$ and $d_{\alpha} \rightarrow\left(d_{a}, \bar{d}_{\dot{a}}\right)$ where $a, \dot{a}=1, \ldots, 8$, and the $\mathrm{SO}(9,1)$ vectors will be decomposed as $X^{m} \rightarrow\left(X^{j}, X^{+}, X^{-}\right)$for $j=1, \ldots, 8$. The precise conventions of this $\mathrm{SO}(8)$ decomposition are discussed in appendix A, where the $\mathrm{SO}(9,1)$ gamma-matrices are expressed in terms of the $\mathrm{SO}(8)$ Pauli matrices $\sigma_{a \dot{a}}^{i}$. In terms of these Pauli matrices, the $D=10$ pure spinor constraint $\lambda^{\alpha} \gamma_{\alpha \beta}^{m} \lambda^{\beta}=0$ takes the form

$$
\lambda_{a} \lambda_{a}=0, \bar{\lambda}_{\dot{a}} \bar{\lambda}_{\dot{a}}=0, \lambda^{a} \sigma_{a \dot{a}}^{i} \bar{\lambda}^{\dot{a}}=0
$$




\subsection{Similarity transformation}

Since the cohomology of $(2.2)$ is described by the $\mathcal{N}=1 D=10$ super Yang-Mills superfield, the BRST operator must impose the Siegel constraint $P^{m}\left(\gamma^{+} \gamma_{m} d\right)_{a}=0$, which is the generator of 8 independent kappa symmetries [9] in the Green-Schwarz formalism. To see that, first note that we can make the constraint explicit in $Q$ by performing a similarity transformation generated by

$$
R=i \frac{P^{i} \bar{N}_{i}}{P^{+}}
$$

where

$$
\bar{N}^{i}=-\frac{1}{\sqrt{2}}\left(\lambda^{a} \sigma_{a \dot{a}}^{i} \bar{\omega}^{\dot{a}}\right)
$$

Observe that

$$
\begin{aligned}
Q^{\prime} & \equiv e^{R} Q e^{-R} \\
& =\lambda^{a} G_{a}+\bar{\lambda}^{\dot{a}} \bar{d}_{\dot{a}},
\end{aligned}
$$

where

$$
G_{a} \equiv \frac{P^{m}}{2 P^{+}}\left(\gamma^{+} \gamma_{m} d\right)_{a}=d_{a}-\frac{P_{i}\left(\sigma^{i} \bar{d}\right)_{a}}{P^{+} \sqrt{2}}
$$

and satisfies

$$
\begin{aligned}
\left\{G_{a}, \bar{d}_{\dot{a}}\right\} & =0, \\
\left\{G_{a}, G_{b}\right\} & =-\frac{\eta_{a b}}{\sqrt{2}}\left(\frac{P^{2}}{P^{+}}\right),
\end{aligned}
$$

with $P^{2}=-2 P^{+} P^{-}+P_{i} P^{i}$. It will be important to note that equation (2.11a) implies that nilpotency of $Q_{p}^{\prime}$ does not rely any more on the pure spinor constraint $\lambda^{a} \bar{\lambda}^{\dot{a}} \sigma_{a \dot{a}}^{i}=0$.

The next step in simplifying the BRST operator is to perform the further similarity transformation generated by

$$
\begin{aligned}
\hat{R} & \equiv i \theta^{a}\left(p_{a}-G_{a}\right) \\
& =\frac{i}{\sqrt{2}}\left(\theta^{a} \sigma_{a \dot{a}}^{i} \bar{p}^{\dot{a}}\right) \frac{P_{i}}{P^{+}} .
\end{aligned}
$$

Unlike $R$ of (2.7), $\hat{R}$ does not commute with the supersymmetry generators and transforms the various operators as $\hat{\mathcal{O}} \equiv e^{\hat{R}} \mathcal{O} e^{-\hat{R}}$ :

$$
\begin{aligned}
& \hat{\bar{d}}_{\dot{a}}=\bar{p}_{\dot{a}}-\frac{i}{\sqrt{2}} P^{+} \bar{\theta}_{\dot{a}}, \\
& \hat{G}_{a}=p_{a}+\frac{i \theta_{a}}{2 \sqrt{2}}\left(\frac{P^{2}}{P^{+}}\right), \\
& \hat{\bar{q}}_{\dot{a}}=\bar{p}_{\dot{a}}+\frac{i}{\sqrt{2}} P^{+} \bar{\theta}_{\dot{a}}, \\
& \hat{q}_{a}=p_{a}+\frac{1}{\sqrt{2}}\left(\sigma^{i} \hat{\bar{q}}\right)_{a} \frac{P_{i}}{P^{+}}-\frac{i \theta_{a}}{2 \sqrt{2}}\left(\frac{P^{2}}{P^{+}}\right) .
\end{aligned}
$$


As expected, $\hat{\bar{d}}_{\dot{a}}$ and $\hat{G}_{a}$ are supersymmetric with respect to the transformed supersymmetry generators $\hat{q}_{a}$ and $\hat{\bar{q}}_{\dot{a}}$. After performing this second similarity transformation, the BRST charge $\hat{Q} \equiv e^{\hat{R}} Q^{\prime} e^{-\hat{R}}$ takes the simple form

$$
\hat{Q}=\lambda^{a}\left(p_{a}+\frac{i}{2 \sqrt{2}} \frac{P^{2}}{P^{+}} \theta_{a}\right)+\bar{\lambda}^{\dot{a}}\left(\bar{p}_{\dot{a}}-\frac{i}{\sqrt{2}} P^{+} \bar{\theta}_{\dot{a}}\right),
$$

where the mass-shell constraint $P^{m} P_{m}$ now appears explicitly.

\subsection{Relation with light-cone vertex operators}

Because of the simple form of $\hat{Q}$, it is easy to compute its cohomology and show equivalence to the light-cone vertex operators. Consider a state with momentum $k^{m}$ (assuming $k^{+} \neq 0$ ) which is represented by the ghost-number 1 vertex operator

$$
\hat{U}=\lambda^{a} \hat{A}_{a}+\bar{\lambda}^{\dot{a}} \hat{\bar{A}}_{\dot{a}}
$$

The $\bar{\lambda}_{\dot{a}} \bar{\lambda}_{\dot{b}}$ component of $\hat{Q} \hat{U}=0$ implies

$$
\hat{\bar{D}}_{\dot{a}} \hat{\bar{A}}_{\dot{b}}+\hat{\bar{D}}_{\dot{b}} \hat{\bar{A}}_{\dot{a}}=\eta_{\dot{a} \dot{b}} \Omega
$$

for some superfield $\Omega$, where $\hat{\bar{D}}_{\dot{a}}=\bar{\partial}_{\dot{a}}-\frac{k^{+}}{\sqrt{2}} \bar{\theta}_{\dot{a}}$. The above equation implies that $\hat{\bar{A}}_{\dot{a}}=$ $-\frac{1}{\sqrt{2} k^{+}} \hat{\bar{D}}_{\dot{a}} \Omega$, which can be set to zero by the gauge transformation $\delta \hat{A}_{\alpha}=-\frac{1}{\sqrt{2} k^{+}} \hat{D}_{\alpha} \Omega$.

In the gauge $\hat{\bar{A}}_{\dot{a}}=0$, the $\lambda_{a} \bar{\lambda}_{\dot{a}}$ component of $\hat{Q} \hat{U}=0$ (together with the constraint $\left.\lambda^{a} \bar{\lambda}^{\dot{a}} \sigma_{a \dot{a}}^{i}=0\right)$ implies that

$$
\hat{\bar{D}}_{\dot{a}} \hat{A}_{a}=\hat{A}_{i} \sigma_{a \dot{a}}^{i}
$$

for some superfield $\hat{A}_{i}$. Equation (2.17) is precisely the constraint on the $\mathrm{SO}(8)$-covariant superfield described in [4], and the most general solution is

$$
\hat{A}_{a}=\Phi(\theta) f_{a}(\bar{\theta}) e^{i k \cdot X},
$$

where $\Phi(\theta)$ is a generic scalar function of $\theta_{a}$ and, as shown in [4], $f_{a}(\bar{\theta})$ is a light-cone super-Yang-Mills superfield depending on an $\mathrm{SO}(8)$ vector $a^{j}$ and an $\mathrm{SO}(8)$ chiral spinor $\chi^{a}$, denoting the transverse polarizations of the gluon and gluino. The explicit formula for $f_{a}(\bar{\theta})$ is

$$
\begin{aligned}
f_{a}(\bar{\theta})= & a^{i}\left(\sigma^{l} \bar{\theta}\right)_{a}\left\{\eta_{i l}+\left(\frac{1}{3 !}\right) \tilde{\theta}_{i l}+\left(\frac{1}{5 !}\right) \tilde{\theta}_{i j} \tilde{\theta}_{j l}+\left(\frac{1}{7 !}\right) \tilde{\theta}_{i j} \tilde{\theta}_{j k} \tilde{\theta}_{k l}\right\}+\left(\frac{\sqrt{2}}{k^{+}}\right) \chi_{a} \\
& +\left(\chi \sigma^{i} \bar{\theta}\right)\left(\sigma^{l} \bar{\theta}\right)_{a}\left\{\left(\frac{1}{2 !}\right) \eta_{i l}+\left(\frac{1}{4 !}\right) \tilde{\theta}_{i l}+\left(\frac{1}{6 !}\right) \tilde{\theta}_{i j} \tilde{\theta}_{j l}+\left(\frac{1}{8 !}\right) \tilde{\theta}_{i j} \tilde{\theta}_{j k} \tilde{\theta}_{k l}\right\}
\end{aligned}
$$

where $\tilde{\theta}_{i j} \equiv\left(\frac{k^{+}}{\sqrt{2}}\right) \bar{\theta}_{\dot{a}} \bar{\theta}_{\dot{c}} \sigma_{\dot{a} \dot{c}}^{i j}$.

Finally, the $\lambda^{a} \lambda^{b}$ component of $\hat{Q} \hat{U}=0$ implies that

$$
f_{a}(\bar{\theta}) \hat{D}_{b} \Phi(\theta)+f_{b}(\bar{\theta}) \hat{D}_{a} \Phi(\theta)=\delta_{a b} \Sigma
$$


for some superfield $\Sigma$ where

$$
\hat{D}_{b} \equiv \frac{\partial}{\partial \theta^{b}}+\frac{\sqrt{2}}{4}\left(\frac{k^{m} k_{m}}{k^{+}}\right) \theta_{b}
$$

Equation (2.20) can only be satisfied if $\hat{D}_{a} \Phi=0$. Since $\hat{D}_{a} \hat{D}_{a}=2 \sqrt{2}\left(\frac{k^{m} k_{m}}{k^{+}}\right), \hat{D}_{a} \Phi=0$ implies both that $k^{2}=0$ and that $\frac{\partial}{\partial \theta^{a}} \Phi=0$ (i.e. $\Phi$ is constant). After rescaling the polarizations by the constant $\Phi$, one finally obtains that the states in the ghost-number one cohomology of $\hat{Q}$ are described by

$$
\hat{U}=\lambda^{a} f_{a}(\bar{\theta}) e^{i k \cdot X},
$$

where $f^{a}(\bar{\theta})$ is the $\mathrm{SO}(8)$-covariant light-cone superfield of $(2.19)$ and $k^{m} k_{m}=0$.

States in the cohomology of the original pure spinor BRST operator are directly obtained from $\hat{U}$ by defining

$$
U \equiv e^{-(R+\hat{R})} \hat{U} e^{(R+\hat{R})}=\lambda^{a} f_{a}(\hat{\theta}) e^{i k \cdot X},
$$

where $\hat{\theta}_{\dot{a}} \equiv \bar{\theta}_{\dot{a}}+\frac{k^{i}}{\sqrt{2} k^{+}}\left(\sigma^{i} \theta\right)_{\dot{a}}$. Note that when the state has vanishing transverse momentum, $k^{i}=0$, the similarity transformations $R$ and $\hat{R}$ of (2.7) and (2.12) vanish and

$$
\left.U\right|_{k^{i}=0}=\hat{U}=\lambda^{a} f_{a}(\bar{\theta}) e^{-i k^{+} X^{-}} .
$$

It is easy to verify that $U$ is the usual vertex operator $\lambda^{\alpha} A_{\alpha}(X, \theta)$ in the gauge $\left(\gamma^{+} A\right)_{\dot{a}}=0$.

Now we will proceed to the more intricate case of the superstring.

\section{Superstring}

In this section, we will repeat the analysis done for the superparticle. After reviewing the pure spinor description of the superstring, we will show that the pure spinor BRST charge $Q=\frac{1}{2 \pi i} \oint\left(\lambda^{\alpha} d_{\alpha}\right)$ can be written after a similarity transformation as

$$
\hat{Q}=\frac{1}{2 \pi i} \oint\left[\lambda^{a}\left(p_{a}+\frac{\sqrt{2}}{2} \hat{T} \theta_{a}\right)+\bar{\lambda}^{\dot{a}}\left(\bar{p}_{\dot{a}}+\frac{\sqrt{2}}{2} \partial X^{+} \bar{\theta}_{\dot{a}}\right)\right],
$$

where

$$
\begin{aligned}
\hat{T} \equiv & -\frac{1}{2}\left(\frac{\partial X^{m} \partial X_{m}}{\partial X^{+}}\right)+i\left(\frac{\bar{p}_{\dot{a}} \partial \bar{\theta}_{\dot{a}}}{\partial X^{+}}\right)+\frac{1}{2} \partial\left(\frac{\hat{J}}{\partial X^{+}}\right) \\
& -\left(\frac{i \sqrt{2}}{4}\right) \frac{\hat{\bar{d}}_{\dot{a}} \partial \hat{\bar{d}}_{\dot{a}}}{\left(\partial X^{+}\right)^{2}}-\left(\frac{1}{2}\right) \frac{\left(\partial \ln \left(\partial X^{+}\right)\right)^{2}}{\partial X^{+}}
\end{aligned}
$$

and

$$
\begin{aligned}
\hat{J} & \equiv-\bar{\omega}_{\dot{a}} \bar{\lambda}_{\dot{a}} \\
\hat{\bar{d}}_{\dot{a}} & \equiv \bar{p}_{\dot{a}}+\frac{\sqrt{2}}{2} \partial X^{+} \bar{\theta}_{\dot{a}} .
\end{aligned}
$$


The structure of $\hat{Q}$ for the superstring closely resembles (2.14) for the superparticle, and one can verify that $\hat{Q}$ is nilpotent using the OPE's

$$
\begin{aligned}
\hat{T}(z) \bar{\lambda}_{\dot{a}} \hat{\bar{d}}_{\dot{a}}(y) & \sim \text { regular }, \\
\hat{T}(z) \hat{T}(y) & \sim \text { regular. }
\end{aligned}
$$

The cohomology of $\hat{Q}$ will be shown to reproduce the usual light-cone superstring spectrum where the similarity transformation maps the DDF-like vertex operators of [6] into the 8 transverse bosonic and fermionic operators, $\alpha_{-n}^{j}$ and $\bar{q}_{-n}^{\dot{a}}$, which create massive superstring states from the massless ground state in light-cone gauge.

\subsection{Review of the pure spinor superstring}

The matter (holomorphic) sector of the pure spinor formalism is constructed from the Green-Schwarz-Siegel variables of [10] and is described by the free action

$$
S_{\text {matter }}=\frac{1}{2 \pi} \int d^{2} z\left(\frac{1}{2} \partial X^{m} \bar{\partial} X_{m}+i p_{\beta} \bar{\partial} \theta^{\beta}\right),
$$

and the free field OPE's

$$
\begin{aligned}
X^{m}(z, \bar{z}) X^{n}(y, \bar{y}) & \sim-\eta^{m n} \ln |z-y|^{2}, \\
p_{\alpha}(z) \theta^{\beta}(y) & \sim \frac{i \delta_{\alpha}^{\beta}}{z-y} .
\end{aligned}
$$

The supersymmetry charge is

$$
q_{\alpha}=\frac{1}{2 \pi} \oint\left\{-p_{\alpha}+\frac{1}{2} \partial X^{m}\left(\gamma_{m} \theta\right)_{\alpha}+\frac{i}{24}\left(\theta \gamma^{m} \partial \theta\right)\left(\gamma_{m} \theta\right)_{\alpha}\right\}
$$

satisfying $\left\{q_{\alpha}, q_{\beta}\right\}=P_{m} \gamma_{\alpha \beta}^{m}$, where $P^{m} \equiv \frac{1}{2 \pi} \oint \partial X^{m}$. The usual supersymmetric invariants are

$$
\begin{aligned}
\Pi^{m} & =\partial X^{m}+\frac{i}{2}\left(\theta \gamma^{m} \partial \theta\right) \\
d_{\alpha} & =p_{\alpha}+\frac{1}{2} \partial X^{m}\left(\gamma_{m} \theta\right)_{\alpha}+\frac{i}{8}\left(\theta \gamma^{m} \partial \theta\right)\left(\gamma_{m} \theta\right)_{\alpha},
\end{aligned}
$$

and the OPE's among them are easily computed to be

$$
\begin{aligned}
\Pi^{m}(z) \Pi^{n}(y) & \sim-\frac{\eta^{m n}}{(z-y)^{2}}, \\
d_{\alpha}(z) \Pi^{m}(y) & \sim-\frac{\gamma_{\alpha \beta}^{m} \partial \theta^{\beta}}{(z-y)}, \\
d_{\alpha}(z) d_{\beta}(y) & \sim i \frac{\gamma_{\alpha \beta}^{m} \Pi_{m}}{(z-y)} .
\end{aligned}
$$

The main feature of the formalism is its simple BRST charge, given by

$$
Q=\frac{1}{2 \pi i} \oint\left(\lambda^{\alpha} d_{\alpha}\right)
$$


where $\lambda^{\alpha}$ is the bosonic ghost. Nilpotency of $Q$ is achieved when $\lambda^{\alpha}$ is constrained by $\lambda \gamma^{m} \lambda=0$, the pure spinor condition. The conjugate of $\lambda^{\alpha}$ will be represented by $\omega_{\alpha}$ and they can be described by the Lorentz covariant action

$$
S_{\lambda}=\frac{1}{2 \pi} \int d^{2} z\left(\omega_{\alpha} \bar{\partial} \lambda^{\alpha}\right)
$$

which has the gauge invariance $\delta \omega_{\alpha}=\phi_{m}\left(\gamma^{m} \lambda\right)_{\alpha}$ due to the pure spinor constraint. The gauge invariant quantities are the Lorentz current, $N^{m n}=-\frac{1}{2}\left(\omega \gamma^{m n} \lambda\right)$, the ghost number current, $J=-\omega \lambda$, and the energy-momentum tensor of the ghost sector, $T_{\lambda}=-\omega \partial \lambda$. The pure spinor constraint also implies the classical constraints on the currents:

$$
\begin{aligned}
N^{m n}\left(\gamma_{n} \lambda\right)_{\alpha}+\frac{1}{2} J\left(\gamma^{m} \lambda\right)_{\alpha} & =0 . \\
N_{m n}\left(\gamma^{m n} \partial \lambda\right)^{\alpha}+J \partial \lambda^{\alpha} & =-4 \lambda^{\alpha} T_{\lambda} .
\end{aligned}
$$

The physical open string spectrum is described by the ghost number one cohomology of $Q$. For example, the massless states are described by the unintegrated vertex

$$
U=\lambda^{\alpha} A_{\alpha}(X, \theta)
$$

where $A_{\alpha}$ is a superfield composed of the zero-modes of $\left(X^{m}, \theta^{\alpha}\right)$. Note that

$$
\{Q, U\}=\lambda^{\alpha} \lambda^{\beta} D_{\alpha} A_{\beta},
$$

where

$$
D_{\alpha} \equiv i \partial_{\alpha}-\frac{1}{2}\left(\gamma^{m} \theta\right)_{\alpha} \partial_{m}
$$

with $\partial_{\alpha}=\frac{\partial}{\partial \theta^{\alpha}}, \partial_{m}=\frac{\partial}{\partial X^{m}}$. Since $\lambda^{\alpha}$ is a pure spinor, $\lambda^{\alpha} \lambda^{\beta} \propto \gamma_{m n p q r}^{\alpha \beta}\left(\lambda \gamma^{m n p q r} \lambda\right)$ and $\{Q, U\}=0$ implies the linearized super Yang-Mills equation of motion [11]:

$$
D \gamma^{m n p q r} A=0 .
$$

The integrated version of (3.17) is given by

$$
V=\frac{1}{2 \pi i} \oint\left\{\Pi^{m} A_{m}+i \partial \theta^{\alpha} A_{\alpha}+i d_{\alpha} W^{\alpha}+N^{m n} F_{m n}\right\}
$$

where $A^{m}$ and $W^{\alpha}$ are the super Yang-Mills fields, constrained by

$$
\begin{aligned}
A_{m} & \equiv \frac{1}{8 i}\left(D_{\alpha} \gamma_{m}^{\alpha \beta} A_{\beta}\right), \\
\left(\gamma_{m} W\right)_{\alpha} & \equiv\left(D_{\alpha} A_{m}+\partial_{m} A_{\alpha}\right),
\end{aligned}
$$

and $F_{m n}=\frac{1}{2}\left(\partial_{m} A_{n}-\partial_{n} A_{m}\right)$. 


\subsection{Similarity transformation}

To show that the cohomology of (3.13) describes the light-cone superstring spectrum, it will be convenient to follow the same procedure as in the previous section for the superparticle. The superstring version of the similarity transformation of (2.7) is

$$
R=-\frac{1}{2 \pi i} \oint\left\{\frac{\bar{N}_{i} \Pi^{i}}{\Pi^{+}}\right\}
$$

and transforms $\lambda^{\alpha}$ and $d_{\alpha}$ as

$$
\begin{aligned}
e^{R} \lambda_{a} e^{-R} & =\lambda_{a}, \\
e^{R} \bar{\lambda}_{\dot{a}} e^{-R} & =\bar{\lambda}_{\dot{a}}-\frac{\left(\sigma^{i} \lambda\right)_{\dot{a}} \Pi_{i}}{\sqrt{2} \Pi^{+}}-\left(\frac{\sqrt{2}}{4}\right) \frac{\left(\sigma^{i} \lambda\right)_{\dot{a}} \partial \bar{N}^{i}}{\left(\Pi^{+}\right)^{2}}, \\
e^{R} d_{a} e^{-R} & =d_{a}-\frac{\left(\sigma^{i} \partial \bar{\theta}\right)_{a} \bar{N}_{i}}{\Pi^{+}}-\sqrt{2} \frac{\partial \theta_{a} \bar{N}_{i} \Pi^{i}}{\left(\Pi^{+}\right)^{2}}, \\
e^{R} \bar{d}_{\dot{a}} e^{-R} & =\bar{d}_{\dot{a}}-\frac{\left(\sigma^{i} \partial \theta\right)_{\dot{a}} \bar{N}_{i}}{\Pi^{+}} .
\end{aligned}
$$

Using the above relations together with the properties $\bar{N}_{i}\left(\sigma^{i} \bar{\lambda}\right)_{a}=\sqrt{2} \lambda_{a} \hat{J}$ and $\bar{N}_{i} \bar{N}^{i}=0$, which follow from the $\mathrm{SO}(8)$ decomposition of (3.15), we obtain

$$
\begin{aligned}
Q^{\prime} & \equiv \frac{1}{2 \pi i} \oint e^{R}\left(\lambda^{\alpha} d_{\alpha}\right) e^{-R} \\
& =\frac{1}{2 \pi i} \oint\left\{\lambda^{a}\left(G_{a}-\sqrt{2} \frac{\partial \theta_{a}}{\Pi^{+}} \hat{J}\right)+\bar{\lambda}^{a} \bar{d}_{\dot{a}}-\left(\frac{\sqrt{2}}{4}\right) \frac{\left(\lambda^{a} \sigma_{a \dot{a}}^{i} \bar{d}^{\dot{a}}\right) \partial \bar{N}^{i}}{\left(\Pi^{+}\right)^{2}}\right\}
\end{aligned}
$$

where

$$
G_{a} \equiv d_{a}-\frac{\left(\sigma^{i} \bar{d}\right)_{a}}{\sqrt{2}}\left(\frac{\Pi^{i}}{\Pi^{+}}\right)
$$

Note that although normal-ordering contributions are being ignored in the explicit computations, the only terms that can receive quantum corrections are

$$
\frac{\partial^{2} \theta_{a}}{\Pi^{+}} \text {and } \partial\left(\frac{\partial \theta_{a}}{\Pi^{+}}\right)
$$

and their coefficients can be determined by requiring nilpotency of $Q^{\prime}$.

The term proportional to $\partial \bar{N}^{i}$ in (3.25) did not appear in the superparticle BRST operator of (2.9), however, it can fortunately be removed by performing a second similarity transformation generated by

$$
R^{\prime}=-\frac{\sqrt{2}}{8 \pi} \oint\left\{\frac{\bar{N}_{i}\left(\partial \theta \sigma^{i} \bar{d}\right)}{\left(\Pi^{+}\right)^{2}}\right\} .
$$

After this transformation, the BRST operator $Q^{\prime \prime}=e^{R^{\prime}} Q^{\prime} e^{-R^{\prime}}$ is

$$
Q^{\prime \prime}=\frac{1}{2 \pi i} \oint\left\{\lambda^{a}\left(G_{a}+\partial \theta_{b} H_{a b}-\left(\frac{\sqrt{2}}{2}\right) \frac{\partial \theta_{a} \hat{J}}{\Pi^{+}}\right)+\bar{\lambda}_{\dot{a}} \bar{d}_{\dot{a}}\right\},
$$


where

$$
H_{a b}=-H_{b a}=\left(\frac{i}{4}\right) \frac{\left(\sigma^{i} \bar{d}\right)_{a}\left(\sigma^{i} \bar{d}\right)_{b}}{\left(\Pi^{+}\right)^{2}},
$$

and the possible normal-ordering contributions have the same form of those in (3.27) and can be determined in an analogous manner. Similar to the superparticle BRST charge $Q^{\prime}$ of (2.9), $Q^{\prime \prime}$ of (3.29) is manifestly supersymmetric and is nilpotent without requiring the pure spinor constraint $\left(\lambda \sigma^{i} \bar{\lambda}\right)=0$ because

$$
\left(G_{a}+\partial \theta_{b} H_{a b}-\left(\frac{\sqrt{2}}{2}\right) \frac{\partial \theta_{a} \hat{J}}{\Pi^{+}}\right)(z)\left(\bar{\lambda}_{\dot{a}} \bar{d}_{\dot{a}}\right)(y) \sim \text { regular. }
$$

To reduce $Q^{\prime \prime}$ to the form of $\hat{Q}$ in (3.1), one needs to perform a further similarity transformation which is a generalization of $\hat{R}$ presented in (2.12) for the superparticle. Expanding in powers of $\theta^{a}$, one finds that

$$
\begin{aligned}
\hat{R}= & \frac{1}{2 \pi i} \oint\left\{\frac{i}{\sqrt{2}} \frac{\partial X^{i}}{\partial X^{+}}\left(\theta \sigma^{i} \bar{p}\right)+\frac{\sqrt{2}}{8} \frac{\left(\theta \sigma^{i} \bar{p}\right)\left(\partial \theta \sigma^{i} \bar{\theta}\right)}{\partial X^{+}}\right. \\
& \left.-\frac{\sqrt{2}}{8} \frac{\left(\theta \sigma^{i} \partial \bar{\theta}\right)\left(\theta \sigma^{i} \hat{\bar{d}}\right)}{\partial X^{+}}-\frac{1}{8} \frac{\left(\partial \theta \sigma^{i} \hat{\bar{d}}\right)\left(\theta \sigma^{i} \hat{\bar{d}}\right)}{\left(\partial X^{+}\right)^{2}}+\frac{i}{2 \sqrt{2}}\left(\frac{\theta \partial \theta}{\partial X^{+}}\right) \hat{J}+\ldots\right\}
\end{aligned}
$$

where $\ldots$ denotes terms which are at least cubic order in $\theta^{a}, \theta^{i j}=\theta^{a} \theta^{c} \sigma_{a c}^{i j}$ and

$$
\hat{\bar{d}}_{\dot{a}} \equiv \bar{p}_{\dot{a}}+\frac{\sqrt{2}}{2} \partial X^{+} \bar{\theta}_{\dot{a}} .
$$

The first term in (3.32) is the same as in the superparticle $\hat{R}$ of (2.12) while the second is required to transform the supersymmetry generator $\hat{\bar{q}}_{\dot{a}} \equiv e^{\hat{R}} \bar{q}_{\dot{a}} e^{-\hat{R}}$ to the simple form

$$
\hat{\bar{q}}_{\dot{a}}=-\frac{1}{2 \pi} \oint\left\{\bar{p}_{\dot{a}}-\frac{\sqrt{2}}{2} \partial X^{+} \bar{\theta}_{\dot{a}}\right\} .
$$

The terms in the second line of (3.32) commute with $\hat{\bar{q}}_{\dot{a}}$ and are necessary so that $\hat{Q} \equiv$ $e^{\hat{R}} Q^{\prime \prime} e^{-\hat{R}}$ has at most linear dependence on $\theta^{a}$. Using the explicit terms in (3.32), it was verified up to linear order in $\theta^{a}$ that

$$
\hat{Q}=\frac{1}{2 \pi i} \oint\left[\lambda^{a}\left(p_{a}+\frac{\sqrt{2}}{2} \hat{T} \theta_{a}\right)+\bar{\lambda}^{\dot{a}}\left(\bar{p}_{\dot{a}}+\frac{\sqrt{2}}{2} \partial X^{+} \bar{\theta}_{\dot{a}}\right)\right],
$$

where $\hat{T}$ is defined in $(3.2)$.

\subsection{Relation with light-cone vertex operators}

To compute the cohomology of $\hat{Q}$ of (3.34), note that the zero mode structure of $\hat{Q}$ is the same as in the superparticle $\hat{Q}$ of (2.14), so the superstring ground state describing the massless states is

$$
\hat{U}=\lambda^{a} f_{a}(\bar{\theta}) e^{i k \cdot X}
$$

of (2.22) where $f^{a}(\bar{\theta})$ is the $\mathrm{SO}(8)$-covariant light-cone superfield of (2.19) and $k^{m} k_{m}=0$. 
To construct massive states in the cohomology, first note that the integrated vertex operators

$$
\begin{aligned}
\alpha_{n}^{j} & \equiv \frac{1}{2 \pi i} \oint \partial X^{j} \exp \left(\frac{i n}{k^{+}} X^{+}\right), \\
\bar{q}_{n}^{\dot{a}} & \equiv-\frac{1}{2 \pi} \oint\left(\bar{p}^{\dot{a}}-\frac{\sqrt{2}}{2} \partial X^{+} \bar{\theta}^{\dot{a}}\right) \exp \left(\frac{i n}{k^{+}} X^{+}\right),
\end{aligned}
$$

are in the cohomology of $\hat{Q}$ for any value of $n$. The relation to the usual Laurent modes becomes clear when $X^{+}(z)=-i k^{+} \ln (z)$, i.e. in light-cone gauge where $X^{+}$is the worldsheet time coordinate. In this gauge, $\exp \left(\frac{i n}{k^{+}} X^{+}\right)=z^{n}$ and we recover the usual Laurent expansion.

One interpretation of the integrated vertex operators of (3.36) is as massless integrated vertex operators for the 8 physical polarizations of the gluon and gluino with momenta $p^{j}=$ $p^{+}=0$ and $p^{-}=\frac{n}{k^{+}}$. However, as will be discussed in [6], another interpretation of (3.36) is as DDF-like operators which act on the ground state vertex operator of (3.35) with $k^{i}=0$ to create excited state vertex operators that describe the massive superstring states. If $X^{+}$ in (3.36) is treated as a holomorphic variable with the OPE $X^{+}(z) X^{-}(y) \sim \ln (z-y)$ and $n$ is a positive integer, the contour integral of $\alpha_{-n}^{j}$ and $\bar{q}_{-n}^{\dot{a}}$ around the ground state vertex operator $\hat{U}=\lambda^{a} f_{a}(\bar{\theta}) e^{i k \cdot X}$ will produce the excited state vertex operators

$$
\begin{aligned}
\alpha_{-n}^{j} \hat{U} & =\frac{1}{(n-1) !}\left(\partial^{n} X^{j}+\ldots\right) \lambda^{a} f_{a}(\bar{\theta}) e^{i\left(k^{j} X^{j}-k^{+} X^{-}-\left(k^{-}+\frac{n}{k^{+}}\right) X^{+}\right)}, \\
\bar{q}_{-n}^{\dot{a}} \hat{U} & =\frac{1}{(n-1) !}\left[\partial^{n-1}\left(\bar{p}^{\dot{a}}+\frac{i k^{+}}{n \sqrt{2}} \partial \bar{\theta}^{\dot{a}}\right)+\ldots\right] \lambda^{a} f_{a}(\bar{\theta}) e^{i\left(k^{j} X^{j}-k^{+} X^{-}-\left(k^{-}+\frac{n}{k^{+}}\right) X^{+}\right)},
\end{aligned}
$$

where $\ldots$ denotes terms proportional to derivatives of $X^{+}$. One can similarly act with any number of $\alpha_{-n}^{j}$ and $\bar{q}_{-n}^{\dot{a}}$ operators on the ground state vertex operator to construct the general excited state vertex operator

$$
\prod_{n>0} \prod_{\dot{a}} \prod_{j}\left(\alpha_{-n}^{j}\right)^{N_{n, j}}\left(\bar{q}_{-n}^{\dot{a}}\right)^{N_{n, \dot{a}}} \hat{U} .
$$

Since $\alpha_{-n}^{j}$ and $\bar{q}_{-n}^{\dot{a}}$ commute with $\hat{Q}$, it is clear that the vertex operators of (3.38) are BRST-closed. And it is easy to see they are not BRST-exact since the worldsheet variables $\partial X^{j}$ and $\left(\bar{p}_{\dot{a}}-\frac{\sqrt{2}}{2} \partial X^{+} \bar{\theta}_{\dot{a}}\right)$ only appear in $\hat{Q}$ through $\hat{T}$. Furthermore, one expects that there are no other states in the cohomology of $\hat{Q}$ as the terms $\left(\lambda^{a} p_{a}\right)$ and $\left(\bar{\lambda}^{\dot{a}} \hat{\bar{d}}_{\dot{a}}\right)$ in (3.34) imply that the cohomology is independent of $\left(\theta^{a}, p_{a}\right)$, and depends on $\left(\bar{\theta}_{\dot{a}}, \bar{p}_{\dot{a}}\right)$ only through combinations that anticommute with $\hat{\bar{d}}_{\dot{a}}$. Also, as in bosonic string theory, the dependence on $\left(X^{+}, X^{-}\right)$is completely fixed by $\hat{T}$. So the cohomology of $\hat{Q}$ is expected to be described by the states of (3.38) which are in one-to-one correspondence with the usual light-cone Green-Schwarz states of the superstring spectrum.

Since the original pure spinor BRST operator $Q$ is related to $\hat{Q}$ by $Q=$ $e^{-R-R^{\prime}} e^{-\hat{R}} \hat{Q} e^{\hat{R}} e^{R+R^{\prime}}$, where the similarity transformations $R, R^{\prime}$ and $\hat{R}$ are defined 
in (3.23), (3.28) and (3.32), covariant BRST-invariant vertex operators in the pure spinor formalism can be related to the vertex operators of (3.38) by acting with these same similarity transformations.

To see how this works, it will be useful to interpret $\alpha_{n}^{j}$ and $\bar{q}_{n}^{\dot{a}}$ of (3.36) as integrated vertex operators for a massless gluon and gluino with momenta $p^{j}=p^{+}=0$ and $p^{-}=\frac{n}{k^{+}}$, and to combine them into a super-Yang-Mills vertex operator by contracting them with the gluon and gluino polarization $a_{j}$ and $\bar{\chi}_{\dot{a}}$ as

$$
\hat{V}_{-n} \equiv a_{j} \alpha_{-n}^{j}-i \bar{\chi}_{\dot{a}} \bar{q}_{-n}^{\dot{a}} .
$$

After performing the similarity transformation with $\hat{R}$ of (3.32), one finds (up to terms quadratic in $\theta^{a}$ ) that

$$
V_{-n}^{\prime} \equiv e^{-\hat{R}} \hat{V}_{-n} e^{\hat{R}}=\frac{1}{2 \pi i} \oint\left\{\Pi_{i} A^{i}+\left(i \partial \bar{\theta}_{\dot{a}}+\frac{n}{\sqrt{2} k^{+}} \bar{d}_{\dot{a}}\right) A^{\dot{a}}\right\}
$$

where $D_{a} A_{\dot{a}}=i \sigma_{a \dot{a}}^{j} A_{j}$, with $D_{a}=\partial_{a}-\frac{n}{k^{+} \sqrt{2}} \theta^{a} . A_{\dot{a}}$ is an $\mathrm{SO}(8)$-superfield that depends only on $\theta_{a}$ and $X^{+}$, in an exact parallel to $f_{a}(\bar{\theta}) e^{-i k^{+} X^{-}}$which depends only on $\bar{\theta}_{\dot{a}}$ and $X^{-}$and satisfies the constraint (2.17). See [6] for further details on $A_{\dot{a}}$ and how it emerges in the pure spinor cohomology.

Finally, the gauge fixed version of the integrated massless vertex of (3.21) is obtained by acting with the similarity transformation $R+R^{\prime}$ of (3.23) and (3.28) which transforms $V_{-n}^{\prime}$ into

$$
\begin{aligned}
V_{-n} & \equiv e^{-R-R^{\prime}} V_{-n}^{\prime} e^{R+R^{\prime}} \\
& =\frac{1}{2 \pi i} \oint\left\{\left(\Pi_{i}-i \frac{n}{k^{+}} \bar{N}_{i}\right) A^{i}+\left(i \partial \bar{\theta}_{\dot{a}}+\frac{n}{\sqrt{2} k^{+}} \bar{d}_{\dot{a}}\right) A_{\dot{a}}\right\} .
\end{aligned}
$$

It is straightforward to see that $R^{\prime}$ commutes with $V_{-n}^{\prime}$, and that $R$ is responsible for reintroducing the ghost Lorentz current $\bar{N}_{i}$ in the vertex.

A detailed discussion of the properties of the DDF-like operators (3.41) is presented in [6]. As shown there, the superstring spectrum is obtained by acting with the above operators on the $\mathrm{SO}(8)$-covariant ground state $\left.\hat{U}\right|_{k^{i}=0}$ of (3.35), allowing a systematic description of all massive pure spinor vertex operators in terms of $\mathrm{SO}(8)$ superfields.

\section{Conclusion}

In this paper, the pure spinor BRST operator $Q=\frac{1}{2 \pi i} \oint\left(\lambda^{\alpha} d_{\alpha}\right)$ was mapped by the similarity transformations of $R, R^{\prime}$ and $\hat{R}$ of (3.23), (3.28) and (3.32) into the nilpotent operator

$$
\hat{Q}=\frac{1}{2 \pi i} \oint\left[\lambda^{a}\left(p_{a}+\frac{\sqrt{2}}{2} \hat{T} \theta_{a}\right)+\bar{\lambda}^{\dot{a}} \hat{\bar{d}}_{\dot{a}}\right],
$$

where $\hat{T}$ is defined in (3.2). The cohomology of $\hat{Q}$ is the usual light-cone Green-Schwarz superstring spectrum and is described by the vertex operators

$$
\prod_{n>0} \prod_{\dot{a}} \prod_{j}\left[\partial^{n-1}\left(\bar{p}^{\dot{a}}+\frac{i k^{+}}{n \sqrt{2}} \partial \bar{\theta}^{\dot{a}}\right)+\ldots\right]^{N_{n, \dot{a}}}\left(\partial^{n} X^{j}+\ldots\right)^{N_{n, j}} \lambda^{a} f_{a}(\bar{\theta}) e^{i\left(k^{j} X^{j}-k^{+} X^{-}-\tilde{k}^{-} X^{+}\right)},
$$


where

$$
\tilde{k}^{-}=\frac{1}{k^{+}}\left[\frac{k^{i} k_{i}}{2}+\sum_{n} n\left(N_{n, \dot{a}}+N_{n, j}\right)\right],
$$

$f_{a}(\bar{\theta})$ is the $\mathrm{SO}(8)$-covariant superfield (2.19) of reference [4], and ... involves derivatives of $X^{+}$. Finally, the similarity transformations of $R, R^{\prime}$ and $\hat{R}$ were argued to map the vertex operators of (4.2) into pure spinor BRST-invariant vertex operators constructed using the DDF-operators described in [6].

\section{Acknowledgments}

NB would like to thank CNPq grant 300256/94-9 and FAPESP grants 2009/50639-2 and 2011/11973-4 for partial financial support, and RLJ would like to thank FAPESP grant 2009/17516-4 for financial support.

\section{A $\mathrm{SO}(9,1)$ to $\mathrm{SO}(8)$ decomposition}

Given an $\mathrm{SO}(9,1)$ chiral spinor $\lambda^{\alpha}$ (antichiral $\bar{\lambda}_{\alpha}$ ), one can write down its $\mathrm{SO}(8)$ components through the use of the projectors $P_{I}^{\alpha}$ and $\left(P_{I}^{\alpha}\right)^{-1} \equiv P_{\alpha}^{I}$, where $I$ generically indicates the $\mathrm{SO}(8)$ indices, defined in such a way that

$$
\begin{array}{lll}
\lambda^{\alpha}=P_{a}^{\alpha} \lambda^{a}+P_{\dot{a}}^{\alpha} \lambda^{\dot{a}}, & \lambda^{a}=P_{\alpha}^{a} \lambda^{\alpha}, & \lambda^{\dot{a}}=P_{\alpha}^{\dot{a}} \lambda^{\alpha}, \\
\bar{\lambda}_{\alpha}=P_{\alpha}^{a} \bar{\lambda}_{a}+P_{\alpha}^{\dot{a}} \bar{\lambda}_{\dot{a}}, & \bar{\lambda}_{a}=P_{a}^{\alpha} \bar{\lambda}_{\alpha}, & \bar{\lambda}_{\dot{a}}=P_{\dot{a}}^{\alpha} \bar{\lambda}_{\alpha} .
\end{array}
$$

Being invertible, they satisfy

$$
\begin{aligned}
P_{a}^{\alpha} P_{\alpha}^{b} & =\delta_{a}^{b}, & P_{\dot{a}}^{\alpha} P_{\alpha}^{\dot{b}} & =\delta_{\dot{a}}^{\dot{b}}, \\
P_{a}^{\alpha} P_{\alpha}^{\dot{a}} & =0, & \delta_{\beta}^{\alpha} & =P_{a}^{\alpha} P_{\beta}^{a}+P_{\dot{a}}^{\alpha} P_{\beta}^{\dot{a}},
\end{aligned}
$$

where $a, \dot{a}=1, \ldots, 8$ are the $\mathrm{SO}(8)$ spinorial indices, representing different chiralities.

Note that upper and lower indices in the $\mathrm{SO}(8)$ language do not distinguish chiralities, i.e., one can define a spinorial metric, $\eta_{a b}\left(\eta_{\dot{a} \dot{b}}\right)$, and its inverse, $\eta^{a b}\left(\eta^{\dot{a} \dot{b}}\right)$, such that $\eta_{a c} \eta^{c b}=$ $\delta_{a}^{b}\left(\eta_{\dot{a} \dot{c}} \eta^{\dot{c} \dot{b}}=\delta_{\dot{a}}^{\dot{b}}\right)$ and are responsible for lowering and raising spinorial indices, respectively, acting as charge conjugation. For example, $\left(\sigma^{i}\right)^{\dot{a} a}=\eta^{a b} \eta^{\dot{a} \dot{b}}\left(\sigma^{i}\right)_{b \dot{b}}$.

Using the projectors, one can build a representation for the gamma matrices $\gamma^{m}$ in terms of the 8-dimensional equivalent of the Pauli matrices,

$$
\begin{aligned}
\left(\gamma^{i}\right)^{\alpha \beta} & \equiv\left(\sigma^{i}\right)^{\dot{a} a}\left(P_{\dot{a}}^{\alpha} P_{a}^{\beta}+P_{a}^{\alpha} P_{\dot{a}}^{\beta}\right), & \left(\gamma^{i}\right)_{\alpha \beta} \equiv\left(\sigma^{i}\right)_{a \dot{a}}\left(P_{\alpha}^{a} P_{\beta}^{\dot{a}}+P_{\alpha}^{\dot{a}} P_{\beta}^{a}\right), \\
\left(\gamma^{-}\right)^{\alpha \beta} & \equiv \sqrt{2} \eta^{a b} P_{a}^{\alpha} P_{b}^{\beta}, & \left(\gamma^{-}\right)_{\alpha \beta} \equiv-\sqrt{2} \eta_{\dot{a} \dot{b}} P_{\alpha}^{\dot{a}} P_{\beta}^{\dot{b}}, \\
\left(\gamma^{+}\right)^{\alpha \beta} & \equiv \sqrt{2} \eta^{\dot{a} \dot{b}} P_{\dot{a}}^{\alpha} P_{\dot{b}}^{\beta}, & \left(\gamma^{+}\right)_{\alpha \beta} \equiv-\sqrt{2} \eta_{a b} P_{\alpha}^{a} P_{\beta}^{b},
\end{aligned}
$$


where

$$
\begin{aligned}
& \left(\sigma^{i}\right)_{a \dot{a}}\left(\sigma^{j}\right)^{\dot{a} b}+\left(\sigma^{j}\right)_{a \dot{a}}\left(\sigma^{i}\right)^{\dot{a} b}=2 \eta^{i j} \delta_{a}^{b}, \\
& \left(\sigma^{i}\right)^{\dot{a} a}\left(\sigma^{j}\right)_{a \dot{b}}+\left(\sigma^{j}\right)^{\dot{a} a}\left(\sigma^{i}\right)_{a \dot{b}}=2 \eta^{i j} \delta_{\dot{b}}^{\dot{a}} \text {, } \\
& \left(\sigma^{i}\right)_{a \dot{a}}\left(\sigma_{i}\right)_{c \dot{c}}+\left(\sigma^{i}\right)_{c \dot{a}}\left(\sigma_{i}\right)_{a \dot{c}}=2 \eta_{a c} \eta_{\dot{a} \dot{c}} \\
& \left(\sigma^{i j}\right)^{a}{ }_{b}\left(\sigma^{i j}\right)_{d}^{c}=8 \eta^{a c} \eta_{b d}-8 \delta_{d}^{a} \delta_{b}^{c}, \\
& \left(\sigma^{i j}\right)^{a}{ }_{b}\left(\sigma^{i j}\right)^{\dot{a}}{ }_{\dot{b}}=4 \sigma_{i}^{\dot{a} a} \sigma_{b \dot{b}}^{i}-4 \delta_{b}^{a} \delta_{\dot{b}}^{\dot{a}},
\end{aligned}
$$

and $\eta^{i j}$ is the flat $\mathrm{SO}(8)$ inverse metric, with $i, j=1, \ldots, 8$. As usual, $\eta_{i k} \eta^{k j}=\delta_{i}^{j}$. Note that

$$
\begin{aligned}
\left\{\gamma^{i}, \gamma^{j}\right\} & =2 \eta^{i j}, \\
\left\{\gamma^{+}, \gamma^{-}\right\} & =-2, \\
\left\{\gamma^{ \pm}, \gamma^{i}\right\}=\left\{\gamma^{+}, \gamma^{+}\right\}=\left\{\gamma^{-}, \gamma^{-}\right\} & =0 .
\end{aligned}
$$

Given a $\mathrm{SO}(9,1)$ vector $N^{m}$, the $\mathrm{SO}(8)$ decomposition used in this work is,

$$
N^{ \pm} \equiv \frac{1}{\sqrt{2}}\left(N^{0} \pm N^{9}\right)
$$

and $N^{i}$, with $i=1, \ldots, 8$, stands for the spatial components. Therefore, the scalar product between $N^{m}$ and $P^{m}$ is given by $N^{m} P_{m}=-N^{+} P^{-}-N^{-} P^{+}+N^{i} P_{i}$.

For a rank-2 antisymmetric tensor $N^{m n}$, the $\mathrm{SO}(8)$ components will be represented as

$$
\left\{N^{i j}, N^{i}=N^{-i}, \bar{N}^{i}=N^{+i}, N=N^{+-}\right\} .
$$

Open Access. This article is distributed under the terms of the Creative Commons Attribution License (CC-BY 4.0), which permits any use, distribution and reproduction in any medium, provided the original author(s) and source are credited.

\section{References}

[1] J.H. Schwarz, Superstring Theory, Phys. Rept. 89 (1982) 223 [InSPIRE].

[2] N. Berkovits, Super Poincaré covariant quantization of the superstring, JHEP 04 (2000) 018 [hep-th/0001035] [INSPIRE].

[3] N. Berkovits, Cohomology in the pure spinor formalism for the superstring, JHEP 09 (2000) 046 [hep-th/0006003] [INSPIRE].

[4] L. Brink, M.B. Green and J.H. Schwarz, Ten-dimensional Supersymmetric Yang-Mills Theory With SO(8) - Covariant Light Cone Superfields, Nucl. Phys. B 223 (1983) 125 [INSPIRE].

[5] Y. Aisaka and Y. Kazama, Relating Green-Schwarz and extended pure spinor formalisms by similarity transformation, JHEP 04 (2004) 070 [hep-th/0404141] [INSPIRE].

[6] R.L. Jusinskas, Spectrum generating algebra for the pure spinor superstring, arXiv:1406.1902 [INSPIRE].

[7] P. Mukhopadhyay, DDF construction and D-brane boundary states in pure spinor formalism, JHEP 05 (2006) 055 [hep-th/0512161] [INSPIRE]. 
[8] N. Berkovits, Covariant quantization of the superparticle using pure spinors, JHEP 09 (2001) 016 [hep-th/0105050] [inSPIRE].

[9] W. Siegel, Hidden Local Supersymmetry in the Supersymmetric Particle Action, Phys. Lett. B 128 (1983) 397 [INSPIRE].

[10] W. Siegel, Classical Superstring Mechanics, Nucl. Phys. B 263 (1986) 93 [INSPIRE].

[11] P.S. Howe, Pure spinors lines in superspace and ten-dimensional supersymmetric theories, Phys. Lett. B 258 (1991) 141 [Addendum ibid. B 259 (1991) 511] [INSPIRE]. 